\title{
An innovation in primary mental health services: The MidValley Well-being Service
}

Margaret Pack

Margaret Pack is a Programme Co-ordinator and Lecturer, Allied Mental Health at the Graduate School of Nursing, Midwifery and Health, Victoria University.

\section{Abstract}

In 2004-5 a new primary health organisation (PHO) project was established that aimed to improve access to equitable health care for the residents of three suburban areas of Lower Hutt, Wellington serviced by three medical practices. After an early successful funding tender in the Ministry of Health, PHO funding round, the MidValley PHO Charitable Trust was formed and from there the MidValley Well-being Service was established and developed in 2005-7. Through positive feedback from Ministry of Health at the two-year evaluation, the service was funded for a third year and at the time of writing continues to grow and expand. This article is a reflection of 'lessons learned' in establishing a new PHO initiative in the community mental health services which is seen as accurately attuning and responding to the needs of the local resident population. The author describes the ways in which social work's traditional concerns of social justice, Treaty of Waitangi principles of partnership, protection, self determination and participation, and a holistic approach to health care can assist in the envisioning of new service development under PHO initiatives.

\section{Introduction}

A new strategy was announced that aimed to empower communities to establish their own services to 'improve, maintain and restore their health' (Ministry of Health, 2003 Minimum Requirements for Primary Health Organisations). The point of difference in the promotional material announcing the 'roll-out' of PHO funding to successful tenders was that the charitable trusts supporting their projects were required to demonstrate the involvement of the local geographic community's identified priorities and needs (ibid). A 'tool kit' was made available for implementing the Ministry of Health strategy through local PHO projects using a set of 'minimum requirements'. These national requirements or guidelines on the establishment of local PHO initiatives were designed to reflect the principles of the Treaty of Waitangi of partnership, participation, protection and self-determination. Access to services for under-resourced populations were to be identified in the local plans. Those groups specified in the national guidelines for the establishment of PHO services included 'Maori, Pacific and low income' residents. The funding was to be made available via the district health boards throughout New Zealand, initially for a two-year period with an evaluation to follow to identify a need for continued funding. Projects meeting the minimum criteria were to be offered further funding for the continuation of the PHO services which had been 
established. The acid test of success was that the district health boards needed to be 'satisfied that the PHO's planning, prioritisation and service delivery will contribute to a reduction in health inequalities' (ibid).

\section{PHO mental health services}

In 2004 the funding for the establishment of mental health PHO services was made available to local communities as part of the wider PHO vision and in response to the MaGPie study. The MaGPie study of prevalence of the diagnosis of treatment of mental health conditions in the community through general practitioners and health centres, highlighted the need for service development to support local health centres and general practitioners in their management of those residents presenting with 'mild to moderate' mental health issues. One in three patients who presented to their general practitioners disclosed a diagnosed mental disorder within the previous 12 months (MaGPie Research Group, 2003). The most common presentations were depressive, anxiety and addiction issues. These mental health diagnoses were found to be more prevalent amongst younger patients and they existed alongside the existence of physical health conditions. Depression and anxiety arising from life events was an area that seemed to respond well to care in the community though in the space of 10-minute general practitioner consultation, the need for supporting services was highlighted by the study (MaGPie Research Group, 2003). Services established in conjunction with health centres which aimed to increase the access to and availability of resources to support recovery in a more holistic way was recommended (ibid). The needs of Maori attending primary health care in a later study of patients presenting to general practitioners highlighted that access to services was an issue influencing the management of mental disorders in the community (MaGPie Research Group, 2005). These studies suggested that the establishment of primary mental health initiatives in the community needed to improve access to services and where ever possible to remove barriers to obtaining appropriate treatment and follow-up services.

\section{MidValley PHO's aims and early initiatives}

One of the early successful tenders to obtain funding in the Ministry of Health PHO funding round was the MidValley Access Primary Health Organisation's charitable trust. MidValley PHO has service provider contracts with three medical practices in the Hutt Valley which includes the Naenae, Taita and Stokes Valley Medical Centres. Of its total resident population of approximately 25,000, some 20,119 residents are in the PHO. Within the total resident population of the MidValley PHO area, 31\% self-identify as Maori or Pacific Island. MidValley PHO's objectives are to 'promote health awareness in identified needs areas', 'to achieve measurable and effective health gains' and to 'help to facilitate the greatest access to health services for Maori, Pacific Island and low income earners in the enrolled PHO patient group' (MidValley PHO Business Plan, 2004).

As part of the MidValley PHO plan to improve the access to quality health care, funding was obtained to establish a mental health service to work in collaboration with locally defined needs and to support the work of the health care professionals at the three PHO medical practices. The MidValley Well-being Service was established to complement also the specialist secondary mental health services and the earlier established primary health care initiatives under MidValley PHO which included an outreach nurse, general practitio- 
ner, community worker, a healthy lifestyles programme and prescription subsidy for those patients unable to meet the costs of prescriptions. These initiatives including the prescription subsidy were established under the Ministry of Health's 'services to improve access' funding or (SIA) grant. These were demonstrations of MidValley PHO's concern to improve inequities in the uptake of health services. The challenge was how to establish the service in a user-friendly way whilst meeting the PHO requirements for collecting and reporting meaningful evidence of the quality and effectiveness of the services provided to enable Ministry of Health to evaluate the results of offering the service. In terms of how we would do this in the primary mental health context, the challenges revolved around the need to provide a DSMIV-TR diagnosis for each new referral for accurate reporting to Ministry of Health on the effectiveness of service we were providing whilst acknowledging the socioeconomic origins of many 'mental health' presentations.

\section{The MidValley Well-being Service}

In 2005 I was appointed as Team Leader to establish the mental health arm of the MidValley Access PHO initiative through consultation with the community-appointed committee that had submitted the successful tender to the Ministry of Health for funding to establish a PHO community mental health service. Through the first two-month consultation period, a business plan, service specifications criteria and clinical process were developed. My brief in this community was to establish and provide with a small team, a confidential and free mental health service that improved the access to service for residents who could not afford to use private services and yet did not fit the criteria for eligibility to the hospital psychiatric services.

As a high proportion of MidValley's resident population self-identified as Maori or Pacific Island, and the committee considered that these residents did not have ready access to culturally appropriate services, our 'Well-being' service, was a multi-cultural as well as a multi-disciplinary team. Three full-time staff were appointed with the principle of service users being able to choose the ethnicity of their worker. To fill the position of 'Kai-awhina' a nurse was appointed who worked with traditional Maori healing methods as well as those derived from current nursing practice. A Pacific Peoples' counsellor, a social worker and mental health professional, fluent in two Pacific Island languages and cultures, used narratives from Pacifica to heal. An intake worker or co-ordinator was our administrative support person who had fluency in two cultures. The co-ordinator's role actively liaised with the referrers at the point of intake and through a brokerage model, clarified the needs of clients at our weekly intake meetings with the three of us and our medical director who was an experienced general practitioner. The community arm of the MidValley PHO employed two advocates: a Kai-mahi for Maori who provided a free transport to treatment service, and a Matai from Western Samoa, who helped families experiencing financial hardship to obtain the housing and benefits they needed to live, through advocacy and offering a translation service. As two rival gangs have headquarters in our region, domestic and gang violence was an everyday reality colouring the environment in which we worked so we were mindful of these informal networks operating in the community.

Working with local marae and existing initiatives such as the medical clinics for youth at local colleges were two of the ways we aimed to work in collaboration with locally defined needs. Access to culturally appropriate services and overcoming barriers to accessing services 
were two guiding principles from the consultation committee. Thus service models needed to facilitate greater access so we encouraged referrals from a range of individuals including general practitioners, self-referral and referral from other agencies such as school guidance counsellors. A service that enabled a range of talking therapies and social interventions to be offered was also central to MidValley's original vision. Thus we offered conventional social work and nursing follow up and psycho-educational programmes that included a listener 'talk-back slot' on Pacific Radio on a range of mental health related topics.

As a social worker by training, I recognised that the PHO vision was similar to what I saw as social work's key aims and values. For social workers social engagement and community participation is achieved through a holistic vision of the individual in the context of their total environments - social, cultural, emotional and psychological. I knew that encouraging social connection and the development of social networks in the community is considered an integral part of the social worker's role. Thus, we promoted our service to large gatherings of community ranging in composition from local residents, general practitioners, DHB staff and a variety of community groups. In Aotearoa New Zealand, the social work role has focused upon what is culturally safe and competent practice by acknowledging the role of Maori as Tangata Whenua. Attending to the monocultural attitudes that exist in society and the stigma of mental illness as creating substrata of inequality were other themes in our deliberations on the new service. The community committee renamed our service originally called 'The MidValley Mental Health Service' to 'The MidValley Well-being Service' to reflect the value we placed individually and collectively on the Recovery movement in mental health services. The Recovery vision is that by 2014 'all tangata motuhake in New Zealand have personal power, a valued place in our whanau and communities and services that support us to lead our own recovery' (Mental Health Commission, 2004). How we would operationalise this vision with the local community became a process of continuing discussion with key advisors within the MidValley PHO consultation committee.

Alongside these deliberations I considered the ecological systems approach in social work (Germain, 1991) which focuses on the context of people in their environments. As a social work practitioner, I viewed the presentations of local residents to the service as resulting from a complex interplay of factors that represent the individual interacting with the boundaries of different parts of the 'system'. So, for example, we attended to the individual's stage of life and the various challenges this represented for them, as well as how as individuals they were interacting within families and communities, including a focus on their cultural belief systems, gender, class and social roles.

From a social rights perspective, there was a social imperative to improve access to service as some residents who self-referred to the service were not enrolled in the PHO, so there was an access to service issue that needed to be navigated from the first contact in many instances. Each of us took responsibility for providing full information about the process of enrolment in the PHO and invited the prospective client to contact us once this process was completed or in some cases of high need, provisionally accepted their self-referral whilst the paper work to enrol them in the PHO was in process. Our co-ordinator often assumed the role of advocate with administration staff at local health centres to complete this initial part of the process with clients, some of whom seemed confused by the steps to take to become an enrolled patient in their PHO and unclear of what the possible advantages were to becoming enrolled. Once they were advised of costs and services available upon enrolment, residents were usually 
enthusiastic about joining their $\mathrm{PHO}$, though this was initially seen as being too difficult or complicated without full information about the process and the possible benefits to them.

\section{Eligibility criteria}

In line with the 'tool kit' for Mental Health PHOs, the MidValley Well-being Service aimed to provide free confidential, service of assessment with 1-6 follow-up sessions for enrolled $\mathrm{PHO}$ patients. Enrolled residents could self-refer or be referred by their general practitioners or other treatment provider. The 'mild to moderate' mental health conditions were to be the focus of attention in line with PHO service establishment guidelines. A summary of who was eligible to use the service is below:

- Open to all PHO enrolled patients.

- 'Mild to Moderate' presentations (Magpie Study).

- Anxiety and depression in response to life events.

- Identifiable recent life stressor.

- Not a client of mental health services.

- Youth 13-18 years.

A process of assessment using a standard format and presentation to a multidisciplinary team comprised of myself as team leader, a clinical co-ordinator, social worker, nurse/kaiawhina and general practitioner occurred once a week to develop treatment options and plans to be further explored with the client at follow-up sessions. Those referred who were assessed as requiring more than six follow-up sessions were discussed at case review meetings and a decision made as to whether referral to other agencies seemed appropriate.

As a part of the initial assessment interview two self-administered scales, the PHQ9 and Hospital Anxiety and Depression scale were completed. The same scales were completed at the final session to ascertain if there had been any change in the symptoms of anxiety and depression reported by the client. These tools formed part of our effectiveness measures for reporting that were required in the toolkit for the establishment of new PHO mental health services.

\section{Results from the first five months}

The following is a summary of themes in the uptake and use of the service in the first five months during which we launched and publicised the MidValley Well-being Service:

- GPs are using the service routinely in their consultations.

- 67 referrals to date Nov-March 2006.

- Services to colleges provided.

- Advisory service to DHB.

- Therapy groups for widowed, young mothers with PND established.

- Educational well-being, marae-based programme launched.

- Community liaison and feedback integrated at monthly committee meetings.

The reasons why clients presented to our service included:

- Bereavement. 
- Failing at occupational goals (work stress).

- Young people's issues (decreased performance at college, truancy, leaving home to go flatting).

- Adjustment related to life cycle changes becoming a parent, work stress, work/life balance, pregnancy, menopause.

- Redundancy/retirement.

- Temporary changes in social relationships (for example, empty nest syndrome following children leaving home, loss of partner or romantic relationship).

How to deliver services to diverse groups whilst working in solidarity with the client's identified goals and priorities was an early challenge, as we found that our clients referred by general practitioners were overwhelmingly female and Pakeha in contrast to the local population profile and mental health client population demographics. However, our client demographic data was in line with the demographic characteristics of patients presenting to their general practitioners in the MaGPie Study (MaGPie, 2003).

We discovered that many of the clients we saw, once we assessed their needs with them, seemed to fit into the 'moderate to severe' categories for mental health follow up, thus they needed referral back to secondary services for the level and type of services they needed to remain well in the community. We found some clients disillusioned with their experiences in their contact with secondary mental health services and when long-term or acute mental health conditions were assessed, we found it sometimes quite difficult to link these clients back to the secondary services who had the appropriate services to provide for their ongoing care, for this reason. The stigma of attending a 'mental health service' seemed to colour the request to the general practitioner to attend our service which was seen as being an extension of the general practitioner's consultation and so part of the wider health endeavour.

When acute needs were identified, we also found it difficult to link some clients to the acute and inpatient mental health services being a relative newcomer to the area, our service was known within some but not all branches of the secondary health and mental health services. Promotion of who we were and what we did became an ongoing need to work collaboratively and effectively with the established secondary mental health services regionally.

\section{Summary of early challenges}

Below is a summary of the key themes we faced:

- Service boundary negotiations with DHB, NGO and other PHO mental health providers.

- Keeping it local (MidValley region).

- Educating GPs about who to refer where.

- Knowing MidValley's place in the Hutt.

- Staying true to our vision - improving the quality of life for residents through access to affordable services.

Though an early criterion of success was for MidValley Well-being Service to reduce acute mental presentations in the local resident population, given that our focus was on the 'mild to moderate' group and given the size of our team, this seemed an unrealistic expectation. 
Having the PHO funding before established agreements were reached as to how the service would be established within the wider mental health services, created the need for discussion and ongoing negotiation as to how we would work together. Therefore, by creating a liaison group that met monthly (The Service Development Group), we were able to define and redefine our roles in relation to the boundaries of our service (given our small capacity and resources). This building of positive relationships across services fostered the goodwill of colleagues to work together in the best interests of our clients. It also enabled a sharing of resources for our staff clinical supervision, professional support and training.

\section{Resolutions to 'turf' debates}

There were three committees established for having ongoing discussions about our service development. These were:

- Service Development Group (DHB, PHO mental health services).

- Participation in cross-sector interest groups (e.g. Lower Hutt Leadership and Advisory Group).

- Liaison at point of intake with Intake Co-ordinator, DHB Mental Health, Maori Mental Health, Vibe, CAT Team, DHB social workers.

\section{Summary of client presenting issues}

Some referrals to our service, once assessed, did not fit the 'mild to moderate' category of mental health presentations for whom the MidValley PHO had established the service. Thus some clients were linked into secondary mental health services when regional, acute or long-term needs were assessed. This linking of appropriate services followed a brokerage model however, some clients once seen in their local community wished to remain at MidValley Well-being Service despite the need for a service that we could not provide. The following table identifies the range of presenting issues from the first five months of the MidValley Well-being Service's operation:

Table one. Client presenting issues during the first five months.

Depression issues 37

Anxiety

Anger management issues

Panic attacks

Grief and loss

Post natal depression

Work stress

Total number of clients seen November-March 2006:

67

\section{Conclusions and implications for service establishment and delivery}

The demand from our resident population was for a service that attended to the demoralisation that resulted from low income, unemployment and work stresses alongside the 
short-term therapies for recovery from depression and anxiety. Thus social factors needed our attention, mirroring the findings of the PHO Interim Evaluation Report, 2007.

Some service users solely have (severe) social problems. Models that tried to address both health and social issues provided more utility to services users, but in many cases the complexity of problems required an interdisciplinary team process (p. 238).

The interim PHO Evaluation Report (2007) highlighted the differences between primary and secondary care in terms of the interplay of social and economic variables in client presentations and the need to have a range of 'treatment' options to be available to meet these diverse needs. As a consequence of this finding, the recommendations that joint endeavours between the Ministry of Health and Ministry of Social Development were suggested. The interdepartmental collaboration has implications for the allocation of resources (ibid, p. 238).

Another implication of the differences with the client group in primary mental health care mirroring the MidValley Well-being Service experience was that many of the enrolled client group did not easily fit a DSMIV-TR diagnosis which was a standard requirement for reporting to Ministry of Health. An amalgam of social, health and emotional/psychological issues formed their own gestalt in the majority of cases we saw. Due to the strengths-based social work theories of practice underpinning my social work practice it was important in my view to respect the client's sense of agency to drive their own recovery. Attaching a mental health diagnosis seemed contrary to the ethos of our service. Thus we struggled to find a language that was both acceptable to us as clinicians, to our clients and to fulfil Ministry of Health reporting requirements. As a compromise position we used the DSMIVTR diagnosis of 'adjustment disorder' with depressed or anxious mood as this category was often more accurately descriptive of the client's circumstances and presentation, as it simultaneously acknowledges the social or wider origins of depression and anxiety across various life transitions.

An alternative language and non-psychiatric terminology is needed to describe the breadth of social and lifestyle factors bringing clients to PHO mental health services and to enable practitioners working within them to respect personal agency and rights of the individual whilst fulfilling statutory responsibilities to continue to serve the wider public. Connolly and Ward (2008) in their deliberations about social rights across the life course use the notion of 'competing interests' to describe this dilemma for practice within statutory settings where rights may be upheld in theory but compromised in the operation. They conclude that:

...non-discrimination, democracy, human rights and service user participation and integrity are important values that resonate in codes of practice internationally. Nevertheless, integrating rights-based values into practice is a complex endeavour particularly when the rights of different parties are perceived to be in conflict (Connolly \& Ward, p. 355).

If primary mental health wishes to support the Recovery movement and support the process of destigmatising mental illness whilst promoting mental health in local communities, alternative reporting mechanisms involving quality measures for consumers are also required as well as those designed by practitioners or clinicians. For example, indicators of social distress could be reported by service users using self-assessment tools focusing on lifestyle factors. The Kessler-10 self assessment does this in very practical ways but its focus is more 
on skills for daily living which we thought was inappropriate to use with our client presentations in mental health though we understand this is used within some PHO services. A focus on stress and lifestyle factors would effectively move the focus from depression and anxiety to some of the social and economic factors underlying those conditions that impede a sense of personal agency.

Our informal evaluation of satisfaction of residents using the MidValley Well-being Service after the first year of its implementation was that the service users were overwhelmingly pleased with the service provided and wished that a no cost service remained available locally. This outcome echoes the results from the interim evaluation of PHO mental health services nationally (Primary Health Initiatives Interim Report, Oct 2007). The provision of the MidValley Well-being Service as other PHO-based mental health services establishes the expectation that free or low-cost services offering a range of support services will be available as of right to enrolled residents of that $\mathrm{PHO}$. This raises an ethical issue if funding for these services does not continue for reasons of 'efficiency' or 'effectiveness' defined in various ways by the funding bodies of the services, when these services constitute residents' definitions of what is central to their community's and their individual or family's well-being. Hopefully the experience of MidValley Well-being Service will highlight that primary mental health is meeting a need locally and is, of necessity, 'a work in progress'.

\section{References}

Connolly, M. \& Ward, T. (2008). Navigating human rights across the life course. Child and Family Social Work, 13, 348-356.

Germain C. D. (1991). Human behaviour in the social environment: An ecological view. New York: Columbia University Press.

Minimum Requirements for Primary Health Organisations. November 2001 retrieved 29/07/2005 from http: / www. moh.govt.nz/moh.nsf/wpg_index/Publications-Minimum+Requirements+... Wellington: Ministry of Health.

Business Plan: MidValley Primary Health Organisation Charitable Trust, April 2004.

MaGPie Research Group (2003). The nature and prevalence of psychological problems in New Zealand primary healthcare: A report on Mental Health and General Practice Investigation (MaGPie). New Zealand Medical Journal, 116(1171). http/ / www.nzma.org.nz/journal/116-1171/379/ retrieved July 2005.

MaGPie Research Group. (2005). Mental disorders among Maori attending their general practitioner. Australian and New Zealand Journal of Psychiatry, 39:401-406.

Mental Health Commission. (2004). Our Lives in 2014: A recovery vision from people with experience of mental illness. Wellington: Mental Health Commission.

Ministry of Health. (2003). Primary health organisation service development toolkit for mental health services in primary health care. Wellington: Ministry of Health.

Primary Mental Health Initiatives Interim Report, Oct 2007. Wellington: School of Medicine and Health Science, University of Otago.

Saleebey, D. (Ed.) (2002). The strengths perspective in social work practice. (3rd ed). Boston, MA: Allyn and Bacon. 